\title{
Le juge et la réforme du travail de 2017
}

\section{Sidnei Machado}

\section{(2) OpenEdition}

\section{Journals}

Édition électronique

URL : https://journals.openedition.org/rdctss/1677

DOI : $10.4000 /$ rdctss. 1677

ISSN : 2262-9815

\section{Éditeur}

Centre de droit comparé du travail et de la sécurité sociale

\section{Édition imprimée}

Date de publication : 1 avril 2019

Pagination : 182-183

ISSN : 2117-4350

\section{Référence électronique}

Sidnei Machado, «Le juge et la réforme du travail de 2017 », Revue de droit comparé du travail et de la sécurité sociale [En ligne], 1 | 2019, mis en ligne le 01 novembre 2021, consulté le 13 novembre 2021. URL : http://journals.openedition.org/rdctss/1677 ; DOI : https://doi.org/10.4000/rdctss. 1677

\section{(c) (1) (9)}

Revue de droit comparé du travail et de la sécurité sociale est mise à disposition selon les termes de la Licence Creative Commons Attribution - Pas d'Utilisation Commerciale - Pas de Modification 4.0 International. 


\section{SIDNEI MACHADO}

UNIVERSITÉ FÉdÉRALE DE PARANÁ - UFPR

\section{LE JUGE ET LA RÉFORME DU TRAVAIL DE 2017}

La Loi 13.467 du 13 juillet 2017 est la loi qui a engendré la réforme structurelle la plus approfondie du Droit du travail brésilien depuis les années 1940. Quelques mois plus tôt, entrait en vigueur la Loi 13.429 du 31 mars 2017 qui réglementait l'emploi temporaire et le recours à la sous-traitance. Ces deux mesures constituant la réforme de la Consolidation des lois du travail (CLT) de 1943 visent à moderniser le marché du travail et à lutter contre le chômage et l'informalité. Cependant, ce nouveau cadre réglementaire va clairement dans le sens de la déréglementation et de la flexibilisation des relations de travail, avec des impacts sur la réduction des coûts du travail et une reconfiguration négative de la négociation collective ainsi que de la structuration syndicale. La réforme brésilienne se situe dans le contexte de la double crise économique et institutionnelle traversée par le pays depuis 2016, avec le processus d'impeachment qui a entraîné une réorientation vers des politiques néolibérales, et ouvert la voie à cette réforme radicale du travail. Du point de vue réglementaire, la réforme met en évidence une forte tension entre ses dispositifs et le texte de la Constitution brésilienne de 1988, en particulier avec la structure et le contenu des droits constitutionnels fondamentaux de protection du travail subordonné et des relations de travail collectives. Cette confrontation entre la réforme et la Constitution a créé les conditions d'une vaste et inévitable judiciarisation de la loi de la réforme brésilienne après son entrée en vigueur, avec vingt-neuf actions directes en inconstitutionnalité devant le Tribunal suprême fédéral (STF), qui contestent des points centraux des lois mentionnées, dans l'objectif d'en voir déclarer l'inconstitutionnalité. Le STF n'a pas rendu de conclusion à la suite de l'analyse de la constitutionnalité de toutes les actions en justice, mais il y a déjà des décisions qui révèlent le modèle d'interprétation de la Cour. Cette contribution analyse les fondements de la jurisprudence de 2018 sur la réforme brésilienne à partir de deux points particuliers: le financement des syndicats (I) et la sous-traitance du travail (II).

\section{I - LA JURISPRUDENCE SUR LE FINANCEMENT SYNDICAL}

La Cour constitutionnelle allait-elle invoquer des limites constitutionnelles ou allait-elle valider la loi ; en d'autres termes, la Cour allait-elle jouer le rôle principal dans la résistance à la réforme, ou allait-elle simplement adopter une attitude passive ? L'interprétation de la Cour a commencé à être révélée dans la décision du 28 juin 2018. La Cour a reconnu la constitutionnalité du dispositif de la réforme qui a transformé la contribution syndicale, autrefois obligatoire pour tous les salariés, en une contribution simplement facultative. Dansles faits, le modèle de contribution facultative, dépendant de l'autorisation individuelle préalable du salarié a tari la source de financement syndical. Au Brésil, la contribution syndicale est un des piliers de la structure du syndicat unique. Né et structuré dans le contexte autoritaire de 1940 (Décret-Loi 2.377 du 28.07.1940), intégré à la CLT de 1943, source continuelle de polémique, le modèle syndical de l'État corporatiste a survécu et a été maintenu dans la Constitution de 1988, avec quelques ouvertures démocratiques. Si la contribution syndicale n'a pas permis que se développe un syndicalisme autonome, la Constitution de 1988 a intégré trois caractéristiques du modèle syndical corporatiste: I'unicité syndicale, le pouvoir réglementaire de la justice du travail et la contribution syndicale obligatoire. Le rôle attribué aux syndicats par la Constitution de 1988 est de représenter toutes les catégories de salariés, affiliés ou non, en plus de participer obligatoirement aux négociations collectives (Constitution, article 8, paragraphes III et VI). L'absence de financement a eu des impacts négatifs sur le maintien de l'organisation syndicale et, en conséquence, sur sa représentativité, dans la mesure où la fragilisation financière a miné le pouvoir de négociation de nombreuses unités. Pour que la Cour puisse évaluer la légalité de la réforme, il est indispensable qu'elle 
s'exprime sur la configuration de la réforme au regard du modèle de structure syndicale prévue dans la Constitution. Dans la décision à la majorité de la Cour brésilienne (six votes favorables un contre), la constitutionnalité de la modification législative a été confirmée, en ce que l'exclusion du caractère obligatoire s'appuie sur les principes qui régissent l'autonomie et la liberté syndicale. Selon l'argumentation majoritaire, on ne peut pas admettre que la contribution syndicale soit imposée aux salariés et aux employeurs quand la Constitution établit que personne n'a l'obligation de s'affilier ou de rester affilié à un syndicat. Par ailleurs, une des prémisses de la réforme était de renforcer la négociation collective, en délégant aux protagonistes de la négociation collective (direction d'entreprises et syndicats) un vaste pouvoir normatif, y compris dérogatoire à la réglementation d'État, dans les limites du respect des droits fondamentaux. Ce qui est paradoxal, c'est que l'élargissement de l'espace de la négociation collective s'est accompagné du retrait d'une importante source de financement syndicale, ce qui affaiblit le pouvoir syndical.

\section{LA JURISPRUDENCE SUR LA SOUS-TRAITANCE DU TRAVAIL}

Jusqu'au 31 mars 2017, il n'existait pas au Brésil de loi sur l'externalisation du travail. La jurisprudence imposait des limites à la sous-traitance pour les activités de support de l'employeur. Cependant, avec la réforme légale, la sous-traitance a fait l'objet d'une vaste déréglementation. La loi de 2017 (Loi 13.429/2017) a modifié l'ancienne loi qui réglementait exclusivement le travail temporaire (Loi 6.019/1974), en élargissant son objet pour envisager également la "prestation de services». L'autre loi (Loi 13.467, de 13/07/2017) traitait également de l'externalisation des services, dans l'objectif de remédier aux omissions de la loi précédente. Les textes des deux lois dans leur ensemble, en plus d'élargir les possibilités de recours au contrat temporaire, autorisent la sous-traitance de toutes les activités des entreprises contractantes, sans exception, y compris les activités principales de l'entreprise. Ainsi, depuis 2017, selon la loi brésilienne, toute modalité de cession de main-d'œuvre par des tiers est licite, l'externalisation étant admise sans limite. La loi sur l'externalisation a également fait l'objet de contestations au moyen de deux actions directes en inconstitutionnalité devant le Tribunal suprême fédéral (STF), mais elles n'ont pas encore été examinées. Cependant, le STF a jugé deux affaires anciennes sur le thème de l'externalisation (ADPF 324 et RE 958252) et s'est prononcé sur la légalité de l'externalisation dans toutes les activités de l'entreprise. Les demandes jugées remettaient en question les restrictions imposées par la jurisprudence du Tribunal supérieur du travail (Document de synthèse 331) en vigueur jusqu'à 1993, qui limitait la sous-traitance aux activités-support des entreprises. Dans le conflit entre la jurisprudence et le texte constitutionnel, le STF a laissé entendre que la pratique de l'externalisation est licite à toutes les étapes du processus de production, y compris pour les activités-objet mêmes de l'entreprise. Les arguments de la décision de la Cour sont favorables à la liberté d'embauche des entreprises, considérant que les limitations du recours à la sous-traitance d'origine jurisprudentielle violaient les principes de la libre initiative, de libre concurrence et de sécurité juridique. Ainsi, selon le STF: «l'externalisation ou toute autre forme de division du travail entre personnes juridiques distinctes est licite, indépendamment de l'objet social des entreprises impliquées, en maintenant la responsabilité subsidiaire de l'entreprise contractante». Avec ce prononcé de la Cour brésilienne, des paramètres interprétatifs favorables à la liberté d'embauche ont été fixés avant la loi de 2017. Ce jugement anticipe la ligne interprétative de la Cour sur la constitutionnalité de la réforme, dès lors qu'elle fait sienne la jurisprudence sur la liberté d'embauche, à partir des principes de liberté d'initiative extraits de la Constitution. Les deux décisions ici analysées suffisent à démontrer qu'au Brésil la jurisprudence constitutionnelle adopte une posture de retenue, en n'imposant pas de limites constitutionnelles à la réforme, en omettant d'analyser le sens profond de la réforme. Cette position du STF de 2018 est le résultat d'une inflexion qui s'est produite à la Cour à partir de 2015. 